\title{
Comparison of the TriTrac-R3D accelerometer and a self-report activity diary with heart-rate monitoring for the assessment of energy expenditure in children
}

\author{
G. Rodriguez ${ }^{1}$, L. Béghin ${ }^{2,3}$, L. Michaud ${ }^{2}$, L. A. Moreno ${ }^{4}$, D. Turck ${ }^{2}$ and F. Gottrand ${ }^{2} *$ \\ ${ }^{1}$ Departamento de Pediatría. HCU 'Lozano Blesa', Zaragoza, Spain \\ ${ }^{2}$ Unité de Gastroentérologie, Hépatologie et Nutrition, Clinique de Pédiatrie, Hôpital Jeanne de Flandre, and Faculté de \\ Médecine, Lille, France \\ ${ }^{3}$ Centre d'Investigation Clinique CIC-9301-INSERM-CHU, Hôpital Cardiologique, Lille, France \\ ${ }^{4}$ EU Ciencias de la Salud, Universidad de Zaragoza, Zaragoza, Spain
}

(Received 8 January 2001 - Revised 26 November 2001 - Accepted 19 January 2002)

\begin{abstract}
Determining total energy expenditure (EE) in children under free-living conditions has become of increasingly clinical interest. The aim of this study was to compare three different methods to assess EE triaxial accelerometry (TriTrac-R3D; Professional Products, Division of Reining International, Madison, WI, USA), activity diary and heart-rate (HR) monitoring combined with indirect calorimetry (IC). Twenty non-obese children and adolescents, aged 5.5 to 16.0 years, participated in this study. Results from the three methods were collected simultaneously under free-living conditions during the same $24 \mathrm{~h}$ schoolday period. Neither activity diary (5904 (SD 1756) kJ) nor the TriTrac-R3D (6389 (SD 979) kJ) showed statistical differences in $24 \mathrm{~h}$ total EE compared with HR monitoring (5965 (SD 1911) kJ). When considering different physical activity (PA) periods, compared with HR monitoring, activity diary underestimates total EE during sedentary periods $(P<0 \cdot 001)$ and overestimates total EE and PA-EE during PA periods $(P<0.001)$ because of the high energy cost equivalence of activity levels. The TriTrac-R3D, compared with HR monitoring, shows good agreement for assessing PA-EE during PA periods (mean difference $+0 \cdot 25$ (SD 1.9) $\mathrm{kJ} / \mathrm{min} ; 95 \%$ CI for the bias $-0.08,0 \cdot 58$ ), but underestimates PA-EE and it does not show good precision during sedentary periods $(-0.87$ (SD 1.4) $\mathrm{kJ} / \mathrm{min}$, $P<0.001)$. Correlation between the vector magnitude generated by the TriTrac-R3D accelerometer and EE of activities derived from HR monitoring is high. When compared with the HR method, the TriTrac-R3D and activity diary are not systematically accurate and must be carefully used for the assessment of children's EE depending on the purpose of each study.
\end{abstract}

Energy expenditure: Accelerometry: Activity diary: Heart-rate monitoring: Physical activity

Energy balance problems occur frequently in patients referred to a children's hospital. Failure to thrive, undernutrition and obesity are pathologies which need a correct evaluation at diagnosis and posterior controls. Theoretically, under free-living conditions, a combination of energy intake and energy expenditure (EE) could allow an estimate of energy balance in each subject. Total daily $\mathrm{EE}$ in children and adolescents is made up of resting (EE), diet-induced thermogenesis, physical activity (PA) and growth (Durnin, 1991). Resting EE is 60-70\% total daily except in those children who are involved in high-energy aerobic competitive sports (World Health Organization, 1985) or, in contrast, in children with a disease which increases resting EE such as cystic fibrosis (Turck \& Michaud, 1998). Diet-induced thermogenesis constitutes $5-10 \%$ total daily EE, PA is $25-30 \%$ total daily $\mathrm{EE}$, and growth on a daily basis is too small to measure except in rapidly growing infants.

The measurement of total EE has been a research procedure under laboratory conditions involving the use of cumulative heart-rate (HR) monitoring and indirect calorimetry (IC; ventilated hood system or whole-body method

\footnotetext{
Abbreviations: EE, energy expenditure; HR, heart-rate; IC, indirect calorimetry; MET, metabolic equivalent tasks; PA, physical activity.

* Corresponding author: Professor F. Gottrand, fax +33 3204459 63, email fgottrand@chru-lille.fr
} 
in a chamber) (Schutz \& Deurenberg, 1996). Nowadays, the development of computerized indirect calorimeters, min-by-min HR monitoring, doubly-labelled water $\left({ }^{2} \mathrm{H}_{2}^{18} \mathrm{O}\right)$ and accelerometry techniques have made the measurement of total $\mathrm{EE}$ under free-living conditions and as a clinical tool possible (Gottrand, 1998). The ${ }^{2} \mathrm{H}_{2}^{18} \mathrm{O}$ method, which is accurate during long-term EE determinations under free-living conditions, has two main disadvantages: (1) the high cost of the isotope ${ }^{18} \mathrm{O}$ and of analysis by spectrometry; (2) it gives a mean value of total daily EE for a period of 1-2 weeks, but it is not possible to measure EE during short-term PA periods or to analyse PA behaviours (Schutz \& Deurenberg, 1996; Gottrand, 1998).

HR monitoring is a non-invasive, socially acceptable and accurate method in groups of children (Livingstone et al. 1992). It is based on the observation that when $\mathrm{O}_{2}$ consumption increases is also associated with an increase in HR. This relationship varies among different individuals, so that individual calibration curves must be generated for each subject by calorimetry and HR monitoring, reflecting different intensities of exercise during free-living conditions (Livingstone et al. 1992; Bitar et al. 1995). Accelerometry techniques, especially triaxial accelerometers, were developed to detect body acceleration in the three planes of a space and it has been shown that it is an objective method to distinguish differences in activity levels between and within individuals (Welk \& Corbin, 1995; Westerterp, 1999), but has low sensitivity for sedentary activities in adults (Bouten et al. 1997).

The objective of the present study was to compare measurements of $\mathrm{EE}$ in children under free-living conditions, obtained by three different method: (1) triaxial accelerometry; (2) activity diary; (3) HR monitoring with individual $\mathrm{EE}$ regression equations performed by IC.

\section{Methods}

Subjects

Twenty non-obese children, seven girls and thirteen boys, aged 5.5 to 16.0 years, all volunteers, participated in the current study after the purpose and the objectives were carefully explained (Table 1). All subjects passed a thorough physical and medical examination and were considered not to have any acute conditions (such as infection) known to interfere with EE. Written informed consent was obtained from children and parents, and the study was approved by the Lille University Research Ethical Committee (Comité Consultatif de Protection des Personnes dans la Recherche Biomédicale).

\section{Protocol}

Subjects arrived by car at the Clinical Investigation Centre of Lille University Hospital, Lille, France (CIC-9301INSERM-CHU) at 08.00 hours after a $12 \mathrm{~h}$ fast. Weight and height were first measured. Resting EE, postprandial EE and EE during PA were measured by IC with an adaptation period of $15 \mathrm{~min}$ under the ventilated canopy system before each measurement period. Resting EE was obtained for $30 \mathrm{~min}$ and postprandial $\mathrm{EE}$ after having breakfast. Breakfast provided approximately $20 \%$ daily energy requirements for each child (World Health Organization, 1985 ) and was made up of milk, sugar, fruit juice, butter, jam and bread. EE was determined 2 hours after having breakfast during PA (described later). HR monitoring was obtained during all measurement periods. Results from the morning session were used to perform the individual regressions of HR $v$. EE. After this 'laboratory validation period', on the same day measurement of $24 \mathrm{~h}$ total EE under free-living conditions began. At the same time,

Table 1. Physical characteristics of subjects

\begin{tabular}{|c|c|c|c|c|c|}
\hline Subjects & Sex & Age (years) & Height (m) & Weight (kg) & BMI $\left(\mathrm{kg} / \mathrm{m}^{2}\right)$ \\
\hline 1 & M & $5 \cdot 50$ & 1.120 & $17 \cdot 9$ & $14 \cdot 2$ \\
\hline 2 & $\mathrm{~F}$ & 5.50 & 1.069 & $19 \cdot 4$ & $16 \cdot 9$ \\
\hline 3 & M & 6.25 & $1 \cdot 261$ & $24 \cdot 0$ & $15 \cdot 0$ \\
\hline 4 & M & $6 \cdot 32$ & $1 \cdot 278$ & $27 \cdot 2$ & $16 \cdot 6$ \\
\hline 5 & M & 6.50 & 1.220 & $21 \cdot 2$ & $14 \cdot 2$ \\
\hline 6 & M & 6.72 & $1 \cdot 204$ & 23.5 & $16 \cdot 2$ \\
\hline 7 & $M$ & 8.00 & 1.253 & $25 \cdot 0$ & $15 \cdot 9$ \\
\hline 8 & M & 9.75 & 1.430 & $30 \cdot 9$ & $15 \cdot 1$ \\
\hline 9 & M & 11.50 & 1.421 & $34 \cdot 2$ & $16 \cdot 9$ \\
\hline 10 & $\mathrm{~F}$ & $12 \cdot 00$ & 1.400 & $30 \cdot 1$ & $15 \cdot 3$ \\
\hline 11 & $\mathrm{~F}$ & $13 \cdot 35$ & 1.435 & $35 \cdot 2$ & $17 \cdot 0$ \\
\hline 12 & M & $14 \cdot 00$ & 1.442 & 39.5 & $18 \cdot 9$ \\
\hline 13 & $\mathrm{~F}$ & $14 \cdot 00$ & 1.540 & $41 \cdot 2$ & $17 \cdot 3$ \\
\hline 14 & M & $14 \cdot 16$ & 1.500 & $42 \cdot 3$ & $18 \cdot 8$ \\
\hline 15 & M & 14.32 & 1.485 & $41 \cdot 3$ & $18 \cdot 7$ \\
\hline 16 & M & $14 \cdot 32$ & 1.574 & $48 \cdot 0$ & $19 \cdot 3$ \\
\hline 17 & $\mathrm{~F}$ & 14.90 & 1.460 & $36 \cdot 6$ & $17 \cdot 1$ \\
\hline 18 & $\mathrm{~F}$ & $15 \cdot 00$ & 1.584 & $55 \cdot 0$ & 21.9 \\
\hline 19 & M & $15 \cdot 00$ & 1.520 & $40 \cdot 2$ & $17 \cdot 3$ \\
\hline 20 & $\mathrm{~F}$ & $16 \cdot 00$ & $1 \cdot 701$ & $63 \cdot 7$ & $22 \cdot 0$ \\
\hline Mean (SD) & & $11.15(3.8)$ & $1.394(0.166)$ & $34.8(12.0)$ & $17 \cdot 2(2 \cdot 2)$ \\
\hline
\end{tabular}

M, male; F, Female. 
each child wore the triaxial accelerometer (TriTrac-R3D; Professional Products, Division of Reining International, Madison, WI, USA) and a HR recorder Holter 24-48 h recorder (synesis, ELA medical, Montrouge, France) for a $24 \mathrm{~h}$ period, carefully writing down each different activity and its duration in the activity diary. The free-living measurement day was chosen to be a usual school day.

\section{Resting energy expenditure and postprandial energy expenditure by indirect calorimetry}

EE was measured by an indirect open-circuit ventilated hood system, Deltatrac II (Datex Instrumentation Corporation, Helsinki, Finland). The RER was calibrated by alcoholburning tests every 6 months according to the calorimeter's manufacturers (Datex Instrumentation Corporation). The calorimeter's manufacturer's certified a CV $<2 \%$ for each measurement of $V_{\mathrm{CO}_{2}}, V_{\mathrm{O}_{2}}$ flows and the RER. Before each measurement, gas analysers were calibrated with a reference gas mixture $\left(\mathrm{CO}_{2}-\mathrm{O}_{2}, 5: 95 \mathrm{v} / \mathrm{v}\right.$, Datex Instrumentation Corporation), and the flow transducer was tested by injecting a known standard volume repeatedly (Matarese, 1997). Expired $\mathrm{CO}_{2}\left(V_{\mathrm{CO}_{2}}\right)$ and inspired $\mathrm{O}_{2}\left(V_{\mathrm{O}_{2}}\right)$ flows were recorded as well as the RER. EE was calculated min-by-min from $\mathrm{O}_{2}$ consumption $\left(V_{\mathrm{O}_{2}}\right.$, $\mathrm{ml} / \mathrm{min}$ ) and $\mathrm{CO}_{2}$ production $\left(V_{\mathrm{CO}_{2}} \mathrm{ml} / \mathrm{min}\right)$ using the Weir (1949) formula without protein correction: $\mathrm{EE}(\mathrm{kJ})=$ $\left(3.9 \times V_{\mathrm{O}_{2}}+1.1 \times V_{\mathrm{CO}_{2}} \times 6.02\right)$. After an adaptation period of $15 \mathrm{~min}$, continuous respiratory exchange measurements were initiated. During resting EE and postprandial EE measurements, children rested quietly in a bed watching videotapes. Only steady-state values were taken into account for analysis (data with a CV $<10 \%$ for both $V_{\mathrm{O}_{2}}$ and dilution air flow, and $<5 \%$ for RER).

\section{Energy expenditure during physical activity period by indirect calorimetry}

EE during PA was determined by IC for consecutive levels of calibrated physical exercise consisting of cycling on a cycloergometer (CARE 910, Bobigny, France), beginning at $0 \mathrm{~W}$ and increasing the braking force ever $30 \mathrm{~s}, 2.5 \mathrm{~W}$ for children aged $<12$ years and $5 \mathrm{~W}$ for children aged $>12$ years, in order to obtain maximal HR close to the theoretical maximal heart frequency $(220$ - age (years)). The seat height, handlebars and pedal crank of the cyclergometer were adjusted to the child's size. The pedalling rate was imposed by the cyclergometer screen at 50 rotations/ $\min$.

\section{Heart-rate monitoring}

The heart-rate recorder (ELH medical) had two channels of electrocardiographic data. This recorder has an event marker and a continuous digital time display that were used to note the different times of the study and to synchronize $H R$ and EE data. HR values defined as the mean of the HR recorded ever min over the specific time periods were calculated with the SYNETEC software (version 1.1; ELA medical).
Individual regression equations of heart-rate $v$. energy expenditure and measurement of total energy expenditure by heart-rate monitoring

Results for HR and EE, recorded simultaneously during laboratory periods (at rest and during postprandial and PA periods), were used to calculate individual polynomial third-order non-linear regression equations (HR v. EE) as described by Bitar et al. (1995). EE and HR were recorded by techniques explained earlier.

Total EE measured by heart-rate monitoring (total $\mathrm{EE}_{\mathrm{HR}}$ ) under free-living conditions was derived from the min-by-min recorded HR by reference to the subject's regression equation for EE $v$. HR. PA-EE by HR monitoring $\left(\mathrm{PA}-\mathrm{EE}_{\mathrm{HR}}\right)$ was calculated with the formula:

$$
\begin{aligned}
\mathrm{PA}-\mathrm{EE}_{\mathrm{HR}}(\mathrm{kJ} / \mathrm{min})= & \text { total } \mathrm{EE}_{\mathrm{HR}}(\mathrm{kJ} / \mathrm{min}) \\
& - \text { resting } \mathrm{EE}(\mathrm{kJ} / \mathrm{min}),
\end{aligned}
$$

where resting EE value is obtained by IC. During the night, $\mathrm{HR}$ is often lower than in resting periods and $\mathrm{HR}-\mathrm{EE}$ equations overestimate night-time EE compared with IC. We therefore used the formula resting $\mathrm{EE}$ - resting EE/ 10 to compute night-time $\mathrm{EE}$ in children (Beghin et al. 2000).

\section{Total energy expenditure by accelerometry}

Total EE measured by triaxial accelerometry (total $\mathrm{EE}_{\mathrm{T}}$ ) under free-living conditions was obtained with the TriTrac-R3D (Professional Products, Madison, WI, USA). The device weighs $170 \mathrm{~g}$ and measures $12 \times 6.5 \times 2.2 \mathrm{~cm}$. It measures acceleration in three individual planes and integrates acceleration to yield one value called 'vector magnitude' (square root of the sum squared of activity counts in each vector). The formula used to convert the 'vector magnitude' to EE from PA is a proprietary formula which will not be released by the manufacturer. Total $\mathrm{EE}_{\mathrm{T}}(\mathrm{kJ} / \mathrm{min})$ is calculated at 1 min intervals by TriTrac-R3D software with the 'vector magnitude' and subject's age, sex, height and weight (Hemokinetics Inc, 1993; Westerterp, 1999). The TriTrac-R3D was worm firmly attached to the anterior torso of the subject at the level of the waist, perpendicular to the mid-line of the anterior thigh.

\section{Total energy expenditure by activity diary}

Children and participating parents were instructed to keep detailed self-reports on the types of behaviours and the times at which these active behaviours began and ended during the $24 \mathrm{~h}$ period when accelerometer and HR monitoring equipment were worn. Upon return of the selfreport sheets, they were reviewed by a trained staff member and discrepancies were resolved with the parent and child. Only activities recorded for duration of $10 \mathrm{~min}$ or more were considered in the comparsion analyses between methods. Activity was converted to multiples of resting metabolic rate in $\mathrm{kJ} / \mathrm{kg}$ per $\mathrm{h}$ using metabolic equivalent tasks (MET) using values from a compendium of PA (Ainsworth et al. 1993). The questionnaire was scored for the activity categories of sedentary (1.0-2.0 
MET), light-moderate PA (2.5-5.0 MET) and hard PA ( $>5$ MET). PA-EE by activity diary $\left(\mathrm{PA}-\mathrm{EE}_{\mathrm{AD}}\right)$ was calculated with the formula:

$$
\begin{aligned}
\mathrm{PA}-\mathrm{EE}_{\mathrm{AD}}(\mathrm{kJ} / \mathrm{min})= & \text { total } \mathrm{EE}_{\mathrm{AD}}(\mathrm{kJ} / \mathrm{min}) \\
& - \text { resting } \mathrm{EE}_{\mathrm{AD}}(\mathrm{kJ} / \mathrm{min}),
\end{aligned}
$$

where resting $\mathrm{EE}_{\mathrm{AD}}$ is the resting metabolic rate with a corresponding MET value of 1 .

\section{Statistical analysis}

Since duration of different measurement periods varied, with resting periods usually longer than PA periods, mean $\mathrm{EE}$ values were expressed in $\mathrm{kJ} / \mathrm{min}$ to avoid the influence of period duration and to allow comparison of results. Results are expressed as mean values and standard deviations. Paired $t$ tests were used to compare mean differences between the TriTrac-R3D self-reported activity and HR monitoring EE during measuring periods. A linear regression analysis was used to examine the relationship between EE derived from the TriTrac-R3D, self-reported activity and HR monitoring measurements. Agreement between methods for determining EE was assessed using the Bland \& Altman (1986) method. The lack of agreement between methods can be evaluated by calculating the bias estimated by the mean difference, standard deviation of the difference and CI for the bias. Correlations were tested using the Spearman test with significance set at $P<0.05$ Statistical analyses were performed using SPSS (version 7.0 for Windows, SPSS, Chicago, IL, USA).

\section{Results}

Total $\mathrm{EE}_{\mathrm{HR}}$ under $24 \mathrm{~h}$ free-living conditions in this group of children was 5965 (SD 1911) kJ/d. There were no statistical differences between total $\mathrm{EE}_{\mathrm{HR}}$ and total $\mathrm{EE}_{\mathrm{T}}$ (6389 (SD 979) kJ/d, $r^{2} 0.73$ ) but the mean difference total $\mathrm{EE}_{\mathrm{HR}}$ - total $\mathrm{EE}_{\mathrm{T}}$ was -424 (SD 1184) $\mathrm{kJ} / \mathrm{d}$ (Fig. 1(a)), $95 \%$ CI for the bias was $-978,130$ and the $95 \%$ CI for the lower and upper limit of agreement were $-3753,-1833$ and 984, 2905 respectively. These intervals are wide, reflecting the small sample size and the great variations of differences. Between total $\mathrm{EE}_{\mathrm{HR}}$ and total $\mathrm{EE}_{\mathrm{AD}}\left(5904(\mathrm{SD} 1756) \mathrm{kJ} / \mathrm{d}, r^{2} 0.72\right)$ there were no statistically significant differences, but the mean difference total $\mathrm{EE}_{\mathrm{HR}}$ - total $\mathrm{EE}_{\mathrm{AD}}$ was 58 (SD 1013) kJ/d (Fig. 1(a)), $95 \% \mathrm{CI}$ for the bias was $-414,534$ and the $95 \%$ CI for the lower and upper limit of agreement were -2788 , -1145 and 1265,2908 respectively. These intervals are as wide as those reported in the Fig. 1(a).

Using an activity diary, 402 different periods could be distinguished according to the level of activity, summarizing all twenty subjects' $24 \mathrm{~h}$ period data (122 periods of PA, 258 periods of sedentary activity and twenty sleeping periods). The results of correlation and statistical difference analysis are shown in Table 2. Comparing the activity diary with $\mathrm{HR}$ monitoring, total $\mathrm{EE}_{\mathrm{AD}}$ was significantly higher than total $\mathrm{EE}_{\mathrm{HR}}$ during PA periods, but lower during sedentary periods; when analysing PA-EE, results remained significantly higher during PA periods. In contrast, during sedentary activity periods there were no statistical differences between activity diary and HR monitoring results even though correlation between these two methods remained low $\left(r^{2} 0 \cdot 38\right)$. When comparing total $\mathrm{EE}_{\mathrm{HR}}$ and total $\mathrm{EE}_{\mathrm{T}}$ there were only statistical differences during PA and sleeping periods and the TriTrac-R3D overestimated total EE during these periods. When analysing PA-EE by HR monitoring and the TriTrac-R3D, there were no differences during PA periods, although significant differences were observed during sedentary periods.

In general, compared with EE measurements by HR monitoring, TriTrac-R3D differences were smaller than activity diary differences (Table 2 ). We can summarize

Table 2. Energy expenditure measured by heart-rate monitoring, activity diary and a triaxial accelerometer (TriTrac-R3D $\ddagger) \S$

\begin{tabular}{|c|c|c|c|c|c|c|c|c|c|}
\hline \multirow[t]{2}{*}{ Measurement method... } & \multirow[b]{2}{*}{$n$} & \multicolumn{2}{|c|}{$\begin{array}{l}\text { HR monitoring } \\
(\mathrm{kJ} / \mathrm{min})\end{array}$} & \multicolumn{3}{|c|}{ Activity diary ( $\mathrm{kJ} / \mathrm{min})$} & \multicolumn{3}{|c|}{ TriTrac-R3D (kJ/min) } \\
\hline & & Mean & SD & Mean & SD & $r^{2} \|$ & Mean & SD & $r^{2} \|$ \\
\hline Total EE all periods & 402 & 5.01 & 2.5 & $5 \cdot 35^{\star}$ & $3 \cdot 2$ & 0.84 & $5.09 \dagger$ & $1 \cdot 8$ & 0.93 \\
\hline Total EE physical activity periods & 122 & $5 \cdot 76$ & 2.9 & $7 \cdot 81^{\star \star \star}$ & 4.5 & 0.68 & $6 \cdot 22^{\star}$ & $2 \cdot 3$ & 0.83 \\
\hline Total EE light-moderate physical activity & 102 & 5.47 & $2 \cdot 8$ & $6 \cdot 22^{\star \star \star}$ & $2 \cdot 3$ & 0.83 & $5 \cdot 93^{*}$ & $2 \cdot 1$ & 0.82 \\
\hline Total EE hard physical activity & 20 & $7 \cdot 35$ & $7 \cdot 6$ & $16 \cdot 0^{\star \star \star}$ & $4 \cdot 0$ & 0.73 & $7 \cdot 81 \dagger$ & 3.0 & 0.88 \\
\hline Total EE sedentary periods & 258 & 4.80 & $2 \cdot 1$ & $4 \cdot 43^{\star \star \star}$ & 1.5 & 0.80 & $4.68 \dagger$ & $1 \cdot 1$ & 0.91 \\
\hline Total EE sleeping periods & 20 & 2.96 & 0.6 & $2 \cdot 38^{\star \star \star}$ & 1.0 & 0.66 & $3 \cdot 67^{\star \star *}$ & 0.5 & 0.87 \\
\hline PA-EE all periods & 402 & $2 \cdot 30$ & $2 \cdot 3$ & $3 \cdot 21^{* * *}$ & $3 \cdot 1$ & 0.36 & $1.58^{\star * \star}$ & 1.7 & 0.51 \\
\hline PA-EE physical activity periods & 122 & 3.05 & $2 \cdot 7$ & $5 \cdot 76^{\star * *}$ & $4 \cdot 3$ & 0.54 & $2 \cdot 80 \dagger$ & $2 \cdot 2$ & 0.71 \\
\hline PA-EE light-moderate physical activity & 102 & $2 \cdot 67$ & $2 \cdot 4$ & $4 \cdot 01^{\star \star \star}$ & 1.6 & 0.62 & $2.46 \dagger$ & 1.9 & 0.66 \\
\hline PA-EE hard physical activity & 20 & $4 \cdot 76$ & 3.3 & $14 \cdot 0^{\star \star \star}$ & 3.9 & 0.63 & $4.43 \dagger$ & 2.9 & 0.80 \\
\hline PA-EE sedentary periods & 258 & 1.92 & 1.8 & $1.92 \dagger$ & 0.8 & 0.38 & $1.00^{* * *}$ & 0.9 & 0.54 \\
\hline
\end{tabular}

(Mean values and standard deviations for twenty children and adolescents)

$\mathrm{EE}$, energy expenditure; $\mathrm{HR}$, heart-rate; PA, physical activity.

Mean values were significantly different from those measured by HR monitoring ( $t$ test): ${ }^{*} P<0.05,{ }^{* * *} P<0.001$.

Mean values were not significantly different from those measured by HR monitoring ( $t$ test): $\dagger P>0.05$.

$\ddagger$ Professional Products, Division of Reining International, Madison, WI, USA.

§ For details of subjects and procedures, see Table 1 and p. 624.

$\| r^{2}$ from linear regressions of EE measured by HR monitoring, $v$. EE measured by activity diary or TriTrac-R3D. 


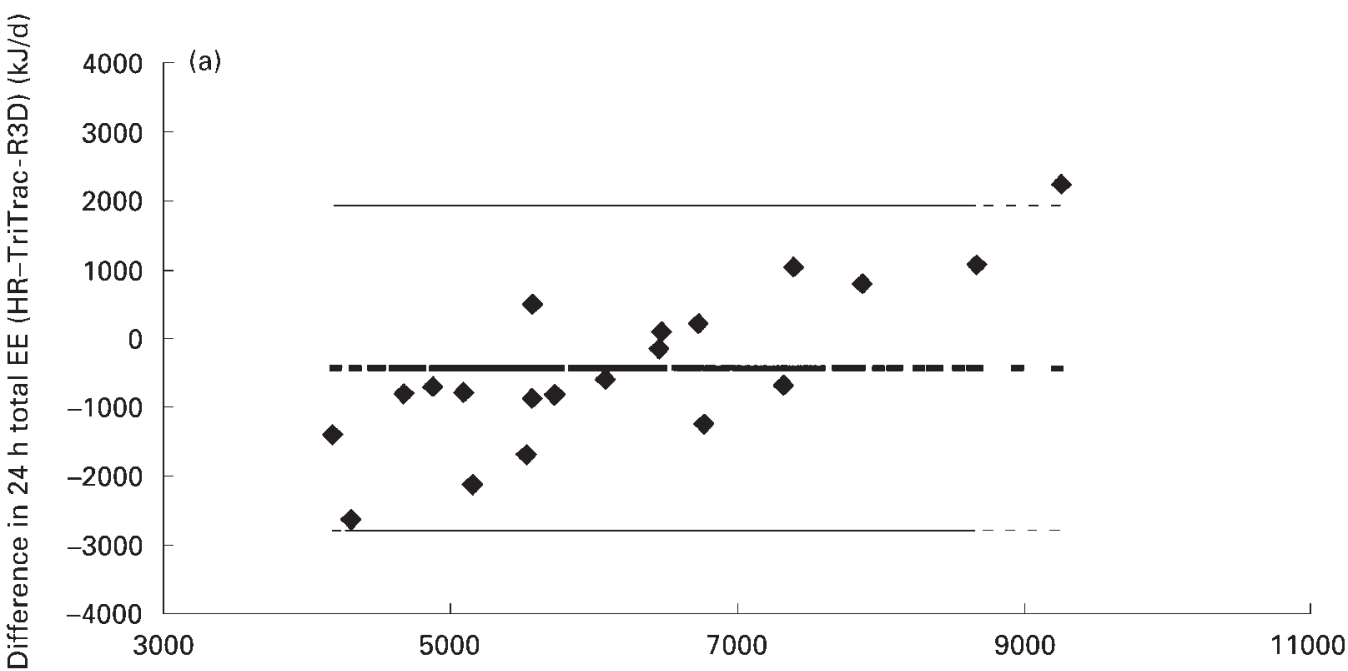

Average $24 \mathrm{~h}$ total EE by HR monitoring and TriTrac-R3D $(\mathrm{kJ} / \mathrm{d})$

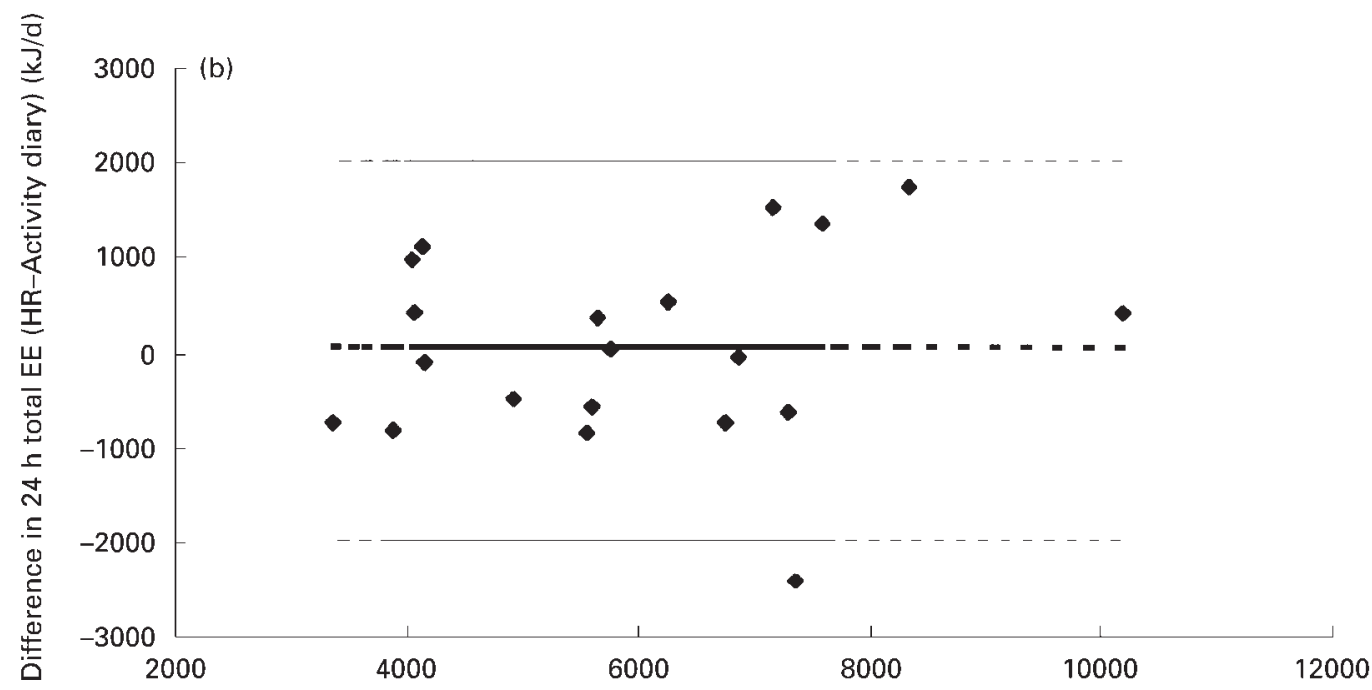

Average $24 \mathrm{~h}$ total EE by HR monitoring and activity diary $(\mathrm{kJ} / \mathrm{d})$

Fig. 1. Difference against mean for $24 \mathrm{~h}$ total energy expenditure (EE) results assessed by (a) heart-rate (HR) monitoring and axial accelerometer (TriTrac-R3D; Professional Products, Division of Reining International, Madison, WI, USA), (b) HR monitoring and activity diary (Bland \& Altman (1986) plots). For details of subjects $(n 20)$ and procedures, see Table 1 and p. 624. ---, Mean value; ---, + or - 2 SD.

the lack of agreement between TriTrac-R3D and HR monitoring PA-EE measurements by calculating the bias, estimated by mean difference and the standard deviation of differences (Table 3). During sedentary periods, PA-EE mean difference was very large $(-0.87$ (SD 1.4) $\mathrm{kJ} / \mathrm{min}$; $95 \%$ CI $0.66,1.04)$. In a Bland-Altman plot, the difference between TriTrac-R3D and HR monitoring against mean PA-EE during all measurement periods is represented in Fig. 2. The scatter of the differences increases as mean PA-EE increases and then, agreement would be wider apart than necessary for small PA-EE. In contrast, when a logarithmic transformation was used to avoid a possible relation between the differences and the mean PA-EE, results showed better agreement for the higher mean values (Fig. 3). In addition, we found the best agreement between PA-EE data during PA periods with a mean difference PA-EE $\mathrm{HR}_{\mathrm{HR}}-\mathrm{PA}-\mathrm{EE}_{\mathrm{T}}$ of 0.25 (SD 1.9) $\mathrm{kJ} / \mathrm{min}$, the $95 \%$ CI for the bias was $-0.08,0.58$ and the $95 \%$ CI for the lower and upper limit of agreement were $-4 \cdot 3,-3 \cdot 1$ and $3 \cdot 6,4 \cdot 8$ respectively (Fig. 4). Correlation between the vector magnitude generated by the TriTracR3D accelerometer and PA-EE of all activities derived from $\mathrm{HR}$ in each children is high: $r^{2}$ ranged from 0.51 to 0.90 ( $P<0.001$, Spearman correlation $)$.

\section{Discussion}

Assessment of EE in human subjects under free-living conditions is limited to very few practical methods. The ${ }^{2} \mathrm{H}_{2}^{18} \mathrm{O}$ method is considered accurate for long-term EE determinations but it is expensive and total EE components cannot be measured (Schutz \& Deurenberg, 1996; 


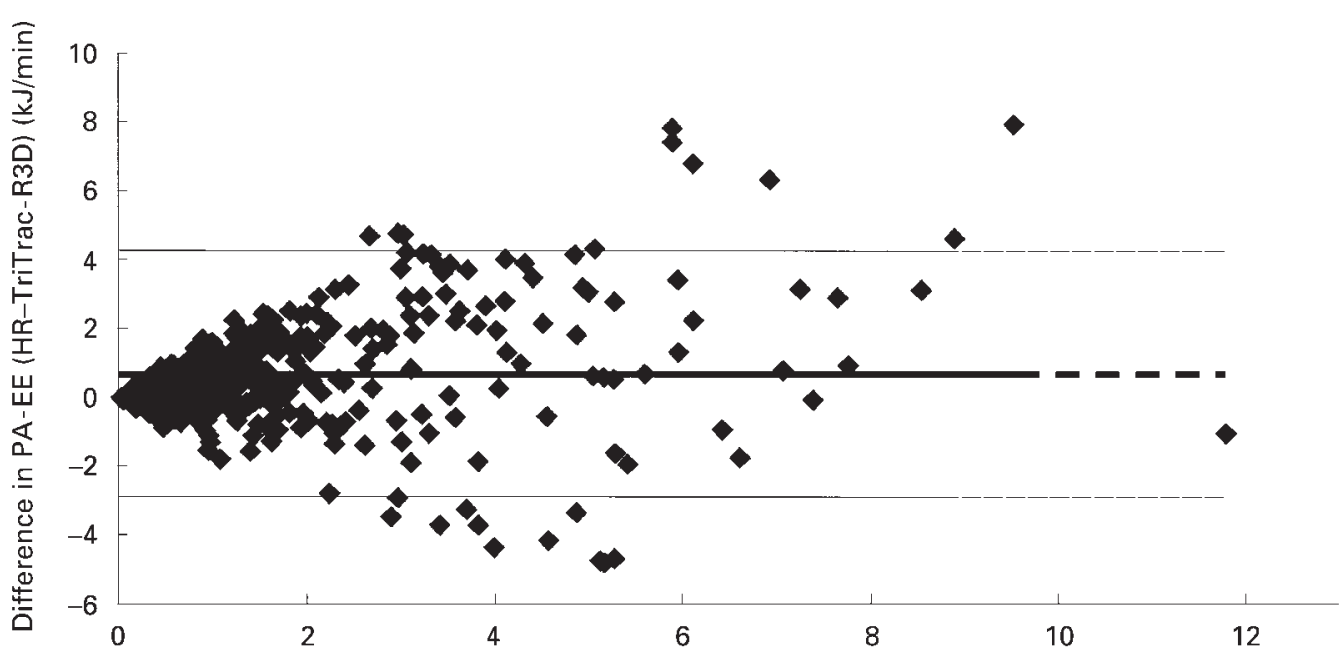

Average PA-EE by HR monitoring and TriTrac-R3D during all measurement periods ( $\mathrm{kJ} / \mathrm{min})$

Fig. 2. Difference against mean for physical activity (PA) energy expenditure (EE) results during all measurement periods assessed by heartrate (HR) monitoring and axial accelerometer (TriTrac-R3D; Professional Products, Division of Reining International, Madison, WI, USA) (Bland \& Altman (1986) plot). For details of subjects $(n 20)$ and procedures, see Table 1 and p. 624. ---, Mean value; ---, + or -2 SD.

Gottrand, 1998). In contrast, HR monitoring allows a close estimation of total EE and its components when an individual regression equation of HR $v$. EE is determined previously. This method is inexpensive, objective, simple and popular with subjects. HR monitoring is appropriate and accurate for predicting group estimates of habitual total EE and levels of PA in free-living adults and children (Livingstone et al. 1992; McCrory et al. 1997). For calculation of individual prediction equations of EE from HR, we have used in the present study a single third-order polynomial relationship which has shown the best fit in children (Bitar et al. 1995; Beghin et al. 2000).

Because of the disadvantages of the reference methods in the assessment of total EE or PA-EE, especially in young children, other less accurate methods are used under free-living conditions (Schutz \& Deurenberg, 1996; Gottrand, 1998). Self-report activity diaries have been found to provide close estimation of total $\mathrm{EE}$ in adolescents and children compared with the results of ${ }^{2} \mathrm{H}_{2}^{18} \mathrm{O}$ or physical working capacity by HR (Bouchard et al. 1983; Bratteby et al. 1997a), and it has also been used to compare EE and daily levels of PA between different groups of population (Sallis, 1991; Bratteby et al. 1997b). As well as the activity diary, a new research instrument, the TriTrac-R3D activity monitor (Professional products), has recently been developed and has already shown very high correlation with HR monitoring during free-play situations in children (Welk \& Corbin, 1995). The TriTracR3D can be used to distinguish differences in activity levels between and within individuals (Westerterp, 1999), but it has limitations in quantifying EE (Jakicic et al. 1999). The reliability of estimates of EE using the TriTrac-R3D has also been tested in adults using indirect calorimetry as reference (Jakicic et al. 1999; Nichols et al. 1999). Inter-instrument and intra-instrument reliability studies show a significant correlation (ranging from 0.73 to 0.92 ) and differences were not significant during various modes and intensities of exercise (Nichols et al. 1999).

Our present study shows total EE and PA-EE results during different level periods of PA, measured using the TriTrac-R3D, self-reporting activity diary and HR

Table 3. Physical activity energy expenditure bias of the triaxial accelerometer (TriTrac-R3D*) compared with heart-rate monitoring $\dagger$

\begin{tabular}{|c|c|c|c|c|}
\hline & \multirow[b]{2}{*}{$n$} & \multicolumn{2}{|c|}{$\begin{array}{c}\text { Mean difference, HR } \\
\text { monitoring }- \text { TriTrac-R3D } \\
(\mathrm{kJ} / \mathrm{min}) \ddagger\end{array}$} & \multirow{2}{*}{$\begin{array}{l}95 \% \mathrm{Cl} \text { for the } \\
\text { bias (kJ/min) }\end{array}$} \\
\hline & & Mean & SD & \\
\hline PA-EE all periods & 402 & 0.66 & 1.7 & $0.45,0.83$ \\
\hline PA-EE physical activity periods & 122 & 0.25 & 1.9 & $-0.08,0.58$ \\
\hline PA-EE light-moderate physical activity & 102 & 0.25 & 1.9 & $-0.12,2 \cdot 42$ \\
\hline PA-EE hard physical activity & 20 & 0.33 & $2 \cdot 3$ & $-0.75,1.42$ \\
\hline PA-EE sedentary periods & 258 & 0.87 & 1.4 & $0.66,1.04$ \\
\hline
\end{tabular}

PA, physical activity; EE, energy expenditure.

* Professional Products, Division of Reining International, Madison, WI, USA.

†For details of subjects and procedures, see Table 1 and p. 624.

$\ddagger$ Mean difference and SD for PA-EE results and $95 \% \mathrm{Cl}$ for the bias as described by Bland \& Altman (1986). 


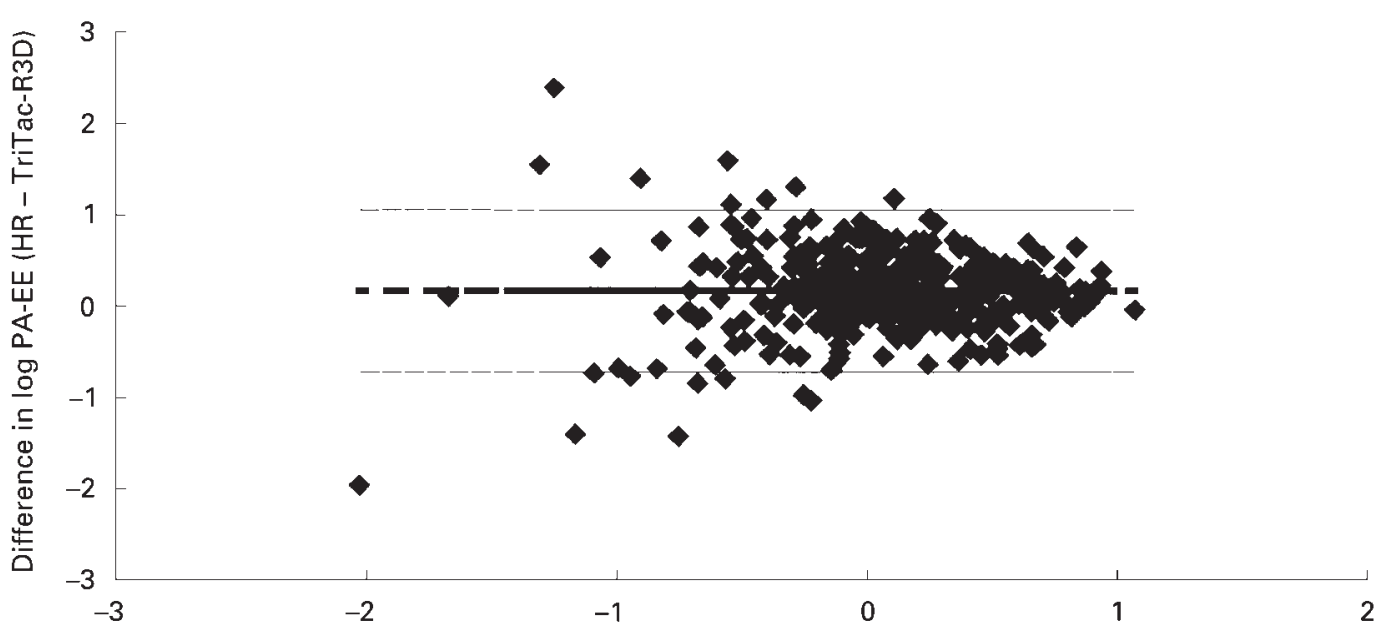

Average log PA-EE by HR monitoring and TriTrac-R3D during all measurement periods

Fig. 3. Difference against mean for physical activity (PA) energy expenditure (EE) results, after logarithmic transformation, during all measurement periods assessed by heart-rate (HR) monitoring and axial accelerometer (TriTrac-R3D; Professional Products, Division of Reining International, Madison, WI, USA) (Bland \& Altman (1986) plot). For details of subjects $(n 20)$ and procedures, see Table 1 and p. 624. ---, Mean value; --- , + or -2 SD. $y=\log 10$ (PA-EE by HR) - log10 (PA-EE by TriTrac-R3D); $x=(\log 10$ (PA-EE by HR) + log10 (PA-EE by TriTrac-R3D))/2.

monitoring performed at the same time, under free-living conditions in the same children population. Considering total daily EE as a whole, the subject sample is probably too limited for a study in children aged $5 \cdot 5$ to $16 \cdot 0$ years. Dividing each measurement day into different PA periods and considering periods of $10 \mathrm{~min}$ or more, 402 different periods with 122 PA periods could be analysed, allowing correlation and comparison between the three methods. Free-living conditions have generated a small group ( $n$ 20) of hard PA periods because an ordinary schoolday was chosen and sustained periods of moderate to vigorous PA are not typical of young people's PA patterns (Armstrong, 1998).
Our self-report activity diary results showed a good correlation with $24 \mathrm{~h}$ total $\mathrm{EE}_{\mathrm{HR}}$ and differences are not significant. Other previous studies have reported the same results compared with ${ }^{2} \mathrm{H}_{2}^{18} \mathrm{O}$ results (Bratteby et al. 1997a). When analysing total EE during different activity periods, activity diary overestimates total EE during PA periods and underestimates total EE during sedentary periods. Total $\mathrm{EE}_{\mathrm{PA}}$ during sleep periods is smaller but PA-EE $E_{P A}$ sedentary values do not present differences; obviously, the cause of the underestimation of total $\mathrm{EE}_{\mathrm{PA}}$ during sedentary periods is an underestimation of resting $\mathrm{EE}_{\mathrm{PA}}$. We have considered that $1 \mathrm{MET}$ of the compendium of physical activities is the resting $\mathrm{EE}_{\mathrm{PA}}$ (Ainsworth et al.

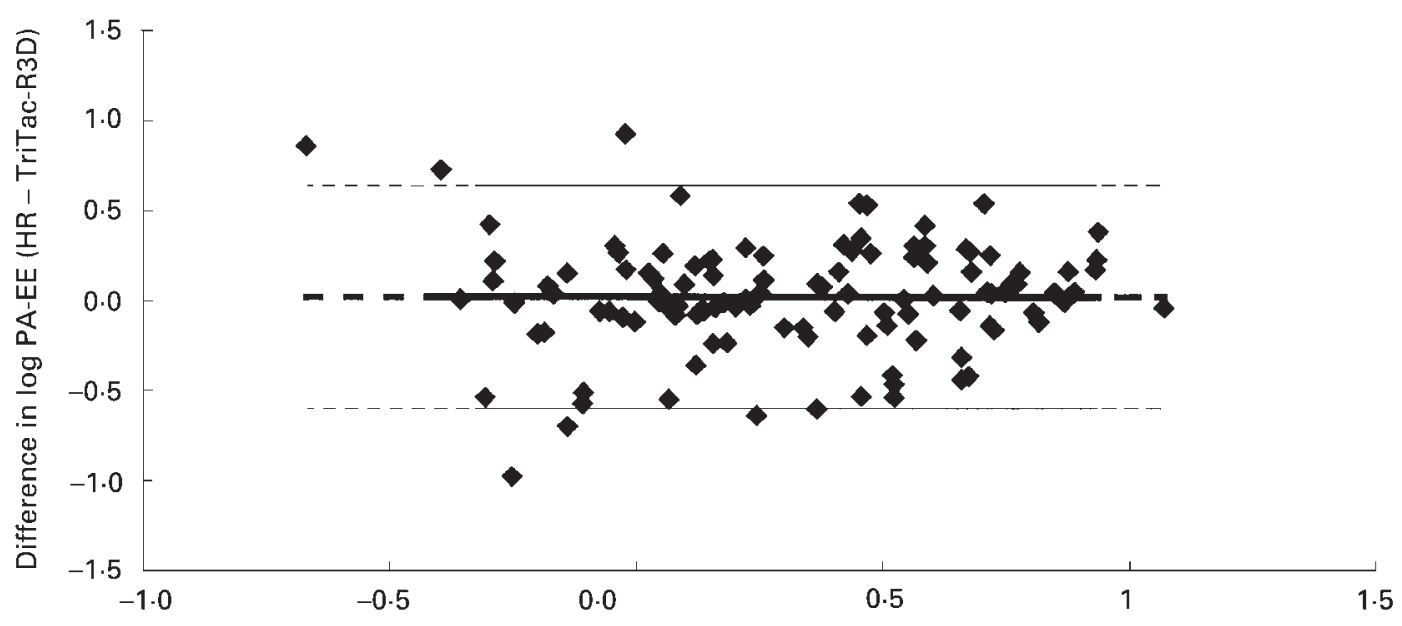

Average log PA-EE by HR monitoring and TriTrac-R3D during physical activity periods

Fig. 4. Difference against mean for physical activity (PA) energy expenditure (EE) results, after logarithmic transformation, during PA periods assessed by heart-rate (HR) monitoring and axial accelerometer (TriTrac-3RD; Professional Products, Division of Reining International, Madison, WI, USA) (Bland \& Altman (1986) plot). For details of subjects $(n 20)$ and procedures, see Table 1 and p. 624. ---, Mean value; ---, + or -2 SD. $y=\log 10$ (PA-EE by HR) $-\log 10$ (PA-EE by TriTrac-R3D); $x=(\log 10$ (PA-EE by HR) $+\log 10$ (PA-EE by HR) + log10 (PA-EE by TriTrac-R3D))/2. 
1993). Similarly, Bouchard et al. (1983) used the same equivalence and the energy cost of resting in bed or sleeping was 1 MET. Bratteby et al. (1997a) improved this latter method with a new denomination: PA ratios (or multiples of resting EE); in that study, resting EE was calculated with a children prediction formula, which avoided resting $\mathrm{EE}_{\mathrm{PA}}$ underestimation, but maintained categorical activity levels similar to Bouchard's classification: resting in bed is 0.95 PA ratio or 0.95 resting EE value, walking outdoors represents 3.3 PA ratio, work of moderate intensity as dancing or cycling $(17-20 \mathrm{~km} / \mathrm{h})$ represent $6.5 \mathrm{PA}$ ratio and running $(10 \mathrm{~km} / \mathrm{h})$ or playing tennis $10 \mathrm{PA}$ ratio. There are at least two factors that could explain the overestimation observed when total $\mathrm{EE}_{\mathrm{AD}}$ and $\mathrm{PA}-\mathrm{EE}_{\mathrm{AD}}$ are compared with HR monitoring results: (1) under freeliving conditions, more than $80 \%$ of children failed to demonstrate a single $10 \mathrm{~min}$ sustained period with HR $\geq 140$ beats/min (Atkins et al. 1997); (2) on revising our total EE data by calorimetry, when children were cycling close to the theoretical maximal heart beat rate, the maximal total EE: resting EE ratio was 4.7 (SD 1.4) PA ratio. This suggests that MET given in the compendium are not well adapted to activities usually accounted in free-living conditions in children. Therefore, an activity diary can be used to compare the time spent at the different categories of PA in population groups and for estimating daily total EE in epidemiological studies (Pols et al. 1997), but not for measuring total EE or PA-EE during specific PA periods.

The accelerometer utilizes the 'vector magnitude' movement count to calculate PA-EE for each min and provides an estimate of resting EE using formulas that were developed for healthy adults. These equations overestimate resting EE by $7 \%$ in children (Bray et al. 1992; Epstein et al. 1996) and the formula used to convert the vector magnitude to EE from PA is a proprietary formula which will not be released by the manufacturer. It is difficult to accept the presentation of results which are based on 'mysterious' calculations that cannot be a matter of discussion. This meant that we have studied in depth, on the one hand, the agreement between the TriTrac-R3D and HR monitoring in the assessment of PA-EE and, on the other hand, the correlation between the real movement variable generated by the accelerometer (vector magnitude) and EE of activities derived from HR monitoring. The results show a good agreement between the two methods in relation with PAEE measurements only during PA periods and high correlation in all subjects between vector magnitude and PA-EE from HR monitoring.

As shown in Tables 2 and 3, TriTrac-R3D significantly underestimates PA-EE during sedentary periods. This discrepancy has already been observed in adults, TriTracR3D underestimates EE compared with the calorimetry method (Jakicic et al. 1999). Shortcomings of this technique are its low sensitivity to sedentary activities and the inability to register static exercise (Bouten et al. 1994, 1997). Another factor that could explain underestimation of EE by the TriTrac-R3D is that diet-induced thermogenesis is not considered with this technique. Diet-induced thermogenesis in children ranges from 5 to $10 \%$ total EE (Durnin, 1991) and during sedentary periods from 16 almost $100 \%$ PA-EE (Bouten et al. 1994). Calorimetry methods can consider diet-induced thermogenesis when individual regression equations of HR $v$. EE are performed, but the accelerometer technique would need a diet-induced thermogenesis correction factor. This would be possible for $24 \mathrm{~h}$ total EE measurements but difficult for different PA period EE measurements in children under free-living conditions.

In summary, there are no statistical differences between $24 \mathrm{~h}$ total EE measured by HR monitoring and activity diary under free-living conditions in children but, when different periods of PA are considered, activity diary underestimates total EE during sedentary periods and overestimates total EE and PA-EE during PA periods because of the high energy cost equivalence of activity levels. Otherwise, although agreement and correlation between TriTrac-R3D and HR monitoring are good during PA periods, TriTrac-R3D cannot be considered as a valid method for the assessment of total EE or PA-EE in children until adapted formulas are developed in further studies. For the assessment of children's EE under free-living conditions, when compared with the HR method, TriTracR3D and the activity diary are not systematically accurate and must be used carefully depending on the purpose of each study.

\section{Acknowledgements}

This study was carried out in the Clinical Investigation Centre of Lille University Hospital (CIC-9301-INSERM$\mathrm{CHU}$ ) and was supported by a grant of the French Ministry of Health (Programme Hospitalier de Recherche Clinique 1997, no. 1901).

\section{References}

Ainsworth BE, Haskell WL, Leon AS, Jacobs DR Jr, Montoye HJ, Sallis JF \& Ptaffenberger RS Jr (1993) Compendium of physical activities: classification of energy costs of human physical activities. Medicine and Science in Sports and Exercise 25, 71-80.

Armstrong N (1998) Young people's physical activity patterns as assessed by heart-rate monitoring. Journal of Sports Sciences 16, S9-S16.

Atkins S, Stratton G, Dugdill L \& Reilly T (1997) The free living physical activity of schoolchildren: A longitudinal study. In Children and Exercise XIX: Promoting Health and WellBeing, pp. 145-150 [N Armstrong, BJ Kirby and JR Welsman, editors]. London: E \& FN Spon.

Beghin L, Budniok T, Vaksman G, Boussard-Delbecque L, Michaud L, Turck D \& Gottrand F (2000) Simplification of the method of assessing daily and nightly energy expenditure in children, using heart-rate monitoring calibrated against open circuit indirect calorimetry. Clinical Nutrition 19, 425435.

Bitar AM, Vermorel M, Fellmann N \& Coudert J (1995) Twentyfour-hour energy expenditure and its components in prepubertal children as determined by whole-body indirect calorimetry and compared with young adults. American Journal of Clinical Nutrition 62, 308-315.

Bland JM \& Altman DG (1986) Statistical methods for assessing agreement between two methods of clinical measurement. Lancet 1, 307-310. 
Bouchard C, Tremblay A, Leblanc C, Lortie G, Savard R \& Thérinault G (1983) A method to assess energy expenditure in children and adults. American Journal of Clinical Nutrition 37, 461-467.

Bouten CV, Koekkoek KTM, Verduin M, Kodde R \& Janssen JD (1997) A triaxial accelerometer and portable data processing unit for the assessment of daily physical activity. IEEE Transactions on Biomedical Engineering 44, 136-147.

Bouten CV, Westerterp KR, Verduin M \& Janssen JD (1994) Assessment of energy expenditure for physical activity using a triaxial accelerometer. Medicine and Science in Sports and Exercise 26, 1516-1523.

Bratteby LE, Sandhagen B, Fan H \& Samuelson G (1997a) A 7day activity diary for assessment of daily energy expenditure validated by the doubly labelled water method in adolescents. European Journal of Clinical Nutrition 51, 585-591.

Bratteby LE, Sandhagen B, Lötborn M \& Samuelson G (1997b) Daily energy expenditure and physical activity assessed by an activity diary in 374 randomly selected 15-year-old adolescents. European Journal of Clinical Nutrition 51, 592-600.

Bray MS, Morrow JR, Pivarnik JM \& Bricker JT (1992) Caltrac validity for estimating caloric expenditure with children. Pediatric Exercise Science 4, 166-179.

Durnin JVGA (1991) Practical estimates of energy requirements. Journal of Nutrition 121, 1907-1913.

Epstein LH, Paluch RA, Coleman KJ, Dominica V \& Anderson K (1996) Determinants of physical activity in obese children assessed by accelerometer and self-report. Medicine and Science in Sports and Exercise 28, 1157-1164.

Gottrand F (1998) Mesure de la dépense énergétique dans les conditions habituelles de vie chez l'enfant (Energy expenditure assessment in children in free living conditions). Archieves de Pédiatrie 5, 1020-1022.

Hemokinetics, Inc. (1993) TriTrac-R3D Research Ergometer Operations. Madison, WI: Hemokinetics, Inc.

Jakicic JM, Winters C, Lagally K, Ho J, Robertson RJ \& Wing RR (1999) The accuracy of the TriTrac-R3D accelerometer to estimate energy expenditure. Medicine and Science in Sports and Exercise 31, 747-754.

Livingstone MBE, Coward WA, Prenctice AM, Davies PSW, Strain JJ, McKenna PG, Mahoney CA, White JA, Stewart
CM \& Kerr MJJ (1992) Daily energy expenditure in freeliving children: comparison of heart-rate monitoring with the $\left({ }^{2} \mathrm{H}_{2}{ }^{18} \mathrm{O}\right)$ doubly labelled water method. American Journal of Clinical Nutrition 56, 343-352.

McCrory MA, Molé PA, Nommsen-Rivers LA \& Dewey KG (1997) Between-day and within-day variability in the relation between heart-rate and oxygen consumption: effect on the estimation of energy expenditure by heart-rate monitoring. American Journal of Clinical Nutrition 66, 18-25.

Matarese LE (1997) Indirect calorimetry: Technical aspects. Journal of the American Dietetic Association 97, Suppl. 2, S154-S160.

Nichols JF, Morgan CG, Sarkin JA, Sallis JF \& Calfas KJ (1999) Validity, reliability, and calibration of the TriTrac accelerometer as a measure of physical activity. Medicine and Science in Sports and Exercise 31, 908-912.

Pols MA, Peeters PHM, Ocké MC, Bueno-de-Mesquita HB, Slimani N, Kemper HCG \& Collette HJA (1997) Relative validity and repeatability of a new questionnaire on physical activity. Preventive Medicine 26, 37-43.

Sallis JF (1991) Self-report measures of children's physical activity. Journal of School Health 61, 215-219.

Schutz Y \& Deurenberg P (1996) Energy metabolism: overview of recent methods used in human studies. Annals of Nutrition and Metabolism 40, 183-193.

Turck D \& Michaud L (1998) Cystic fibrosis: nutritional consequences and management. Baillère's Clinical Gastroenterology 12, 805-822.

Weir JB (1949) New methods for calculating metabolic rate with special reference to protein metabolism. Journal of Physiology 109, $1-9$.

Welk GJ \& Corbin CB (1995) The validity of the TriTrac-R3D activity monitor for the assessment of physical activity in children. Research Quarterly for Exercise and Sport 66, $1-8$.

Westerterp KR (1999) Physical activity assessment with accelerometers. International Journal of Obesity 23, Suppl. 3, S45-S49.

World Health Organization (1985) Energy and Protein Requirements. Report of a Joint FAO/WHO/UNU Expert Consultation. Technical Report Series no. 724 Geneva: WHO. 